\title{
HERNIORRAFIA AMBULATORIAL COMPARADA À CONVENCIONAL
}

\section{AMBULATORY VERSUS CONVENTIONAL HERNIORRAPHY}

\author{
Daniel Nunes e Silva ${ }^{1}$; Márcio Longhi Griebeler²; Sheila Ferreira Fernandes²; \\ Leonardo Quadros da Paixão ${ }^{1}$; Fernando Antônio Bohrer Pitrez, TCBC-RS ${ }^{3}$
}

\begin{abstract}
RESUMO: Objetivo: O tratamento cirúrgico ambulatorial de hérnias inguinais é uma tendência em muitos serviços de saúde. No entanto, em nosso meio, tal procedimento ainda não perfaz uma rotina. Objetivamos analisar os benefícios e complicações da herniorrafia inguinal ambulatorial comparada à convencional. Método: Realizamos uma análise retrospectiva envolvendo, inicialmente, 105 pacientes submetidos a herniorrafia inguinal ambulatorial (HIA) e internação convencional (IC) de fevereiro a outubro de 2002 no Complexo Hospitalar Santa Casa de Porto Alegre (CHSCPA). Foram avaliados os benefícios e complicações no $15^{\circ}, 90^{\circ}$ e $180^{\circ}$ dias de pós-operatório. Além disso, foi realizada avaliação dos custos hospitalares. Os métodos de análise incluíram o teste t de Fischer e $\mathrm{X}^{2}$ . Foram considerados estatisticamente significativos resultados com $\mathrm{p}<0,05$. Resultados: A amostra final constou de 97 pacientes. A taxa total de complicações foi de $36,08 \%$. Não verificamos diferença significativa entre os grupos em quaisquer dos tempos de análise. A complicação mais freqüente foi a dor no local da incisão, sendo referida em $24,7 \%$ dos pacientes. Dentre as outras complicações, observamos equivalência entre os grupos. Quanto aos custos, ressaltamos uma redução de $20 \%$ no valor de cada herniorrafia quando realizada ambulatorialmente. Também observamos uma redução significativa da lista de espera para realização de reparo de hérnias após a instituição do procedimento ambulatorial. Conclusão: A herniorrafia ambulatorial realizada no CHSCPA é um procedimento seguro, não determina maiores complicações quando comparada a herniorrafia convencional. Além disso, facilita a dinâmica hospitalar com custos evidentemente menores (Rev. Col. Bras. Cir. 2004; 31(5): 287-290) - ISSN 0100-6991.
\end{abstract}

Descritores: Herniorrafia; Anestesia local; Hérnia inguinal; Procedimentos cirúrgicos ambulatórios; Complicações.

\section{INTRODUÇÃO}

A cirurgia ambulatorial tem sido introduzida como tratamento ideal para um grande número de pacientes. ${ }^{1} \mathrm{~A}$ herniorrafia é considerada um procedimento de baixo risco e de pequeno porte, assim está justificada a sua realização a nível ambulatorial. A herniorrafia inguinal é uma das cirurgias mais realizadas nos hospitais gerais. ${ }^{2}$ Nos hospitais norteamericanos, cerca de $50 \%$ das herniorrafias inguinais já são realizadas ambulatorialmente. ${ }^{3}$ As vantagens atribuídas a herniorrafia inguinal ambulatorial incluem: redução nos custos hospitalares, retorno precoce do paciente ao domicílio, redução dos índices de infecção e maior disponibilidade de leitos hospitalares. ${ }^{3,4}$ Entretanto, a herniorrafia ambulatorial não perfaz a rotina em nosso meio. ${ }^{5,6} \mathrm{O}$ objetivo deste estudo é avaliar os benefícios da realização da herniorrafia inguinal ambulatorial (HIA) comparativamente à herniorrafia com internação convencional (IC) em termos de morbidade e custos hospitalares.

\section{MÉTODO}

Foram analisados, retrospectivamente, 97 prontuários de pacientes submetidos a herniorrafias inguinais primárias realizadas eletivamente no Complexo Hospitalar Santa Casa de Porto Alegre (CHSCPA), no período de janeiro a julho de 2002. Todos os pacientes foram submetidos a exames pré- operatórios de rotina, sendo então liberados clinicamente para o procedimento cirúrgico. Todos os procedimentos foram realizados pela mesma equipe cirúrgica, seguindo uma rotina previamente estabelecida. As técnicas de reparo herniário seguiram as descrições dos autores originais e incluíram as técnicas de Shouldice, Bassini e Lichtenstein. ${ }^{7}$ A escolha da técnica operatória foi feita no trans-operatório de acordo com as condições da parede abdominal do paciente, reservandose as técnicas de Lichtenstein e Shouldice para os casos mais complicados, com parede abdominal enfraquecida. O reparo herniário foi realizado com fio Prolene 0 e, na técnica de Lichtenstein, foi utilizada tela de polipropileno, com fio Prolene 0 para fixação da tela. Para todos os pacientes, foi realizada a tricotomia da região inguinal imediatamente antes do procedimento, seguida da administração de $1 \mathrm{~g}$ de Cefalotina endovenosa. Os pacientes foram alocados em dois grupos: aqueles submetidos à herniorrafia inguinal com internação convencional (IC), ou seja, permanência intra-hospitalar superior a $24 \mathrm{~h}$, e aqueles submetidos à herniorrafia inguinal ambulatorial (HIA). A técnica anestésica empregada incluiu a raquidiana e a local com sedação. Em três casos, a anestesia raquidiana não foi efetiva, tendo sido realizada anestesia geral.

O anestésico local utilizado consistiu de $20 \mathrm{ml}$ de Lidocaína a 0,5\% (com Adrenalina 1:200.000), $20 \mathrm{ml} \mathrm{de}$ Bupivacaína a 0,25\% (com Adrenalina 1:200.000) e $20 \mathrm{ml}$ de Soro Fisiológico $0,9 \%{ }^{6}$. Foram aplicados $30 \mathrm{ml}$ da solução

1. Residentes de Cirurgia Geral da Fundação Faculdade Federal de Ciências Médicas de Porto Alegre (FFFCMPA).

2. Doutorandos da FFFCMPA.

3. Professor Adjunto de Cirurgia Geral da FFFCMPA, Chefe do Centro de Hérnias do Complexo Hospitalar Santa Casa.

Recebido em 28/08/2003

Aceito para publicação em 29/05/2004

Trabalho realizado no Complexo Hospitalar Santa Casa de Porto Alegre, Fundação Faculdade Federal de Ciências Médicas de Porto Alegre. 
anestésica na espinha ilíaca ântero-superior, no tubérculo púbico, na linha planejada para a incisão cutânea e profundamente à aponeurose do músculo oblíquo externo ${ }^{6}$. No transoperatório, de acordo com a solicitação do paciente, novas doses do anestésico foram infiltradas para o alívio total da dor. Para sedação leve, foram utilizados Midazolam $(5 \mathrm{mg} / \mathrm{ml}$ EV) e Fentanila $(0,05 \mathrm{mg} / \mathrm{ml} \mathrm{EV})$, a critério do anestesista.

A raquianestesia foi realizada com Bupivacaína Pesada $0,5 \%, 10-15 \mathrm{mg}$. Nestes pacientes também utilizamos sedação endovenosa.

Imediatamente antes de deixarem a sala cirúrgica e durante a internação, todos os pacientes receberam Clorofenotiazinilscopina (3ml IM 6/6h) e Cetoprofeno (100mg IM 12/12h). Os mesmos foram orientados a solicitar medicação analgésica de resgate, conforme necessidade, para alívio total da dor. O esquema analgésico aplicado, então, constituiu-se de Morfina 3mg EV 3/3h. No momento da alta, foi prescrito Paracetamol (750mg VO de 6/6h), para uso em caso de dor. Embora o transporte dos pacientes no retorno ao domicílio não tenha sido controlado pela equipe de pesquisa, todos os pacientes foram orientados a utilizar automóvel ou ambulância, na condição de passageiros e a permanecer em repouso por quatro semanas. As consultas ambulatoriais de revisão ocorreram no $15^{\circ}, 90^{\circ}$ e $180^{\circ}$ dia de pós-operatório (PO). Foram avaliados, através de questionários dirigidos e exame físico, a prevalência de complicações (dor, infecção, hematoma, orquite e recidiva). Realizamos também avaliação dos custos hospitalares, baseados na média de permanência hospitalar de cada grupo considerando-se a tabela do Sistema Único de Saúde atualmente vigente. A taxa de complicações foi analisada pelo teste de Pearson no programa estatístico SPSS 10.1. Diferenças foram consideradas significativas se valor de $p$ menor que $0,05(\mathrm{p}<0,05)$.

\section{RESULTADOS}

Dos 97 pacientes incluídos no estudo, 57 (58,8\%) foram submetidos à herniorrafia inguinal com internação convencional (IC) e, o restante, 40 (41,2\%), à herniorrafia ambulatorial. As características da amostra estão expostas na Tabela 1. Não observamos diferença estatística entre os grupos.

Conforme observamos na Tabela 2, o índice total de complicações pós-operatórias foi de $36,08 \%$. A complicação mais freqüente foi a dor no local da incisão, sendo referida em $24,7 \%$ dos pacientes. A infecção na ferida operatória foi diagnosticada num total de oito pacientes $(8,2 \%)$, sendo que

Tabela 1 - Características dos pacientes incluídos no estudo em distribuição por herniorrafia ambulatorial e internação convencional.

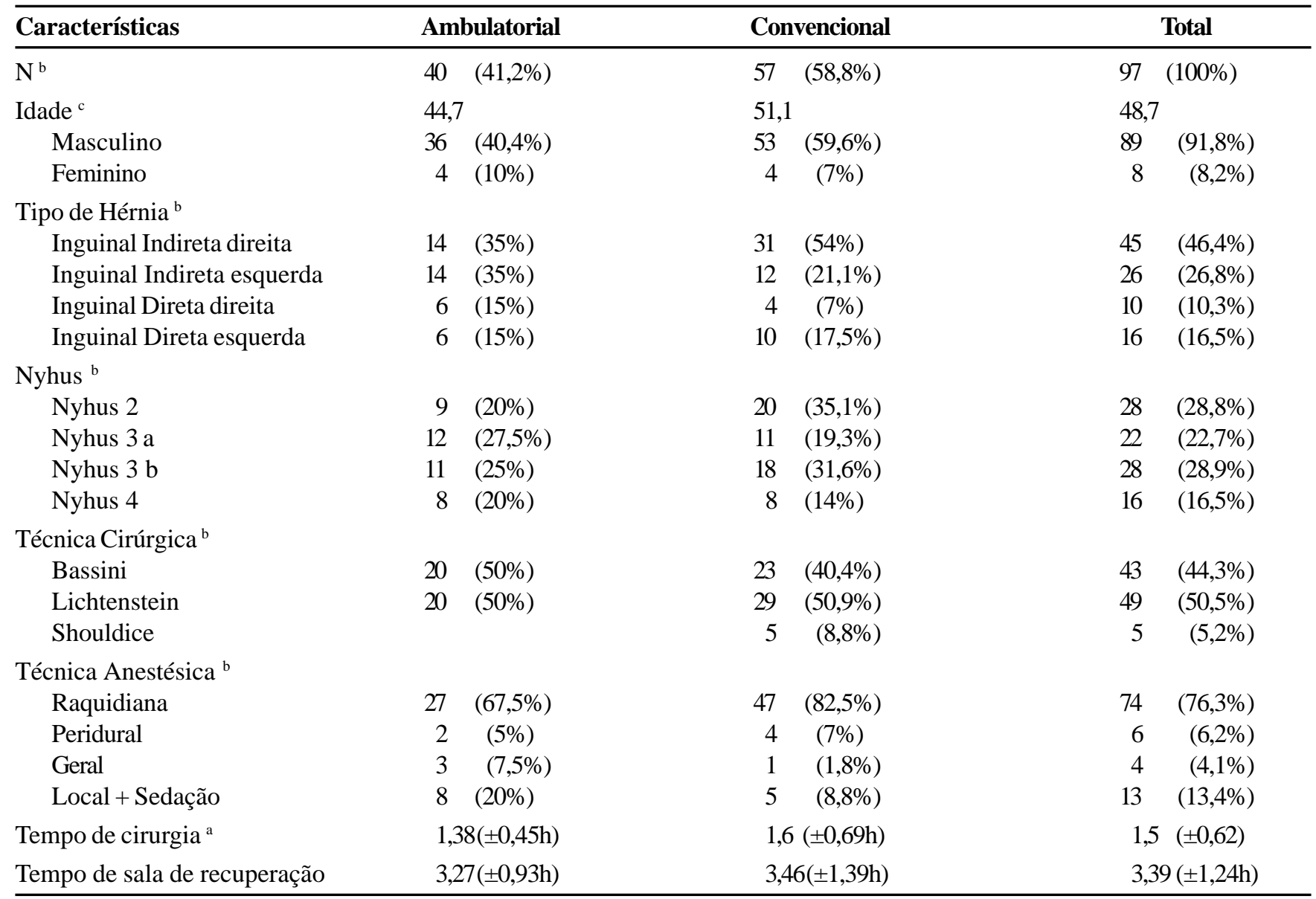

a - dados em média \pm desvio padrão

c- dados em média

$b$ - dados em $N(\%)$

$P>0,05$ para todos os dados nos grupos em questão 
Tabela 2 - Complicações nos períodos de 15, 90 e 180 dias de pós-operatório nos grupos herniorrafia ambulatorial (HIA) e internação convencional (IC).

\begin{tabular}{lccccccc}
\hline Pós-operatório & \multicolumn{2}{c}{$\mathbf{1 5}$ dias de PO } & \multicolumn{2}{c}{$\mathbf{9 0}$ dias de PO } & \multicolumn{2}{c}{ 180 dias de PO } & \multirow{2}{*}{ Total } \\
Complicações & HIA & IC & HIA & IC & HIA & IC & \\
\hline Dor & 4 & 9 & 2 & 5 & 1 & 3 & 24 \\
Infecção & 4 & 4 & - & - & - & - & 8 \\
Hematoma & - & 1 & - & - & - & - & 1 \\
Orquite & - & 1 & - & - & - & - & 1 \\
Recidiva & - & - & - & - & 1 & - & 1 \\
Total & 8 & 15 & 2 & 5 & 2 & 3 & 35 \\
\hline
\end{tabular}

p>0,05 para todas as comparações (tempo e grupo).

todos apresentaram melhora com o tratamento administrado. Verificamos somente um paciente que apresentou hematoma e outro, edema escrotal, com resolução com tratamento conservador. Não verificamos diferença estatisticamente significativa entre os grupos em nenhuma das variáveis avaliadas $(\mathrm{p}>0,05)$. Ocorreu apenas uma recidiva herniária $(1,03 \%)$, em um paciente do grupo da cirurgia ambulatorial, submetido à técnica de Lichtenstein, com necessidade de re-intervenção.

No grupo da internação convencional, a média de internação de 2,67 dias ( $\pm 1,27$ dias). Todos os pacientes do grupo herniorrafia ambulatorial permaneceram, no máximo, 24 horas hospitalizados.

O custo de permanência hospitalar média de cada paciente no grupo da herniorrafia ambulatorial foi de $\mathrm{R} \$ 498,00$ e no grupo da internação convencional foi de $\mathrm{R} \$ 678,60$, perfazendo uma diferença de $26,6 \%$ entre os grupos.

\section{DISCUSSÃO}

A partir de janeiro de 2001, quando foi publicada, no Diário Oficial da União, a Portaria $\mathrm{N}^{\circ} 44^{8}$, autorizando a realização de herniorrafias ambulatoriais em hospitais vinculados ao SUS, o Serviço de Cirurgia Geral do Complexo Hospitalar Santa Casa de Porto Alegre tem preconizado sua realização. A herniorrafia, a nível ambulatorial, justifica-se por ser considerada um procedimento de baixo risco e de pequeno porte.

A herniorrafia ambulatorial pode ser considerada, atualmente, a melhor escolha para mais de $90 \%$ dos reparos herniários eletivos. ${ }^{9}$ Além disso, é considerada segura e custo-efetiva ${ }^{10}$ realizada como padrão em muitos centros de referência em herniorrafias. ${ }^{6}$

Uma das vantagens da herniorrafia ambulatorial é a redução no tempo de espera para realização da cirurgia. De acordo com o Informe do Controle Ambulatorial do Serviço de Cirurgia Geral do CHSCPA, após a instituição da herniorrafia ambulatorial, o número de pacientes em lista de espera cirurgia reduziu-se em média $80 \% .{ }^{11}$ Tal redução pode ser explicada pela menor permanência intra-hospitalar do paciente bem como pela conseqüente maior rotatividade e eficiência, apesar da manutenção ou mesmo redução do número de leitos hospitalares destinados ao atendimento dos pacientes submetidos às herniorrafias.
Não verificamos diferença na taxa de infecção entre os grupos. Verificamos a presença de infecção em $8 \%$ dos pacientes. Índice que pode ser considerado alto, justificado pelas limitações de nossa amostra. Destacamos que a maior permanência intra-hospitalar, em nosso estudo, não resultou em maior taxa de infecção. A antissepsia e a antibioticoprofilaxia foram aplicadas a todos os pacientes, independente da técnica a ser utilizada.

De acordo com o exposto, evidenciamos um índice de recidiva de $1,1 \%$ o que é comparável com os resultados da literatura. Perez et al. ${ }^{12}$ avaliaram 145 pacientes com hérnia inguinal unilateral submetidos a herniorrafia ambulatorial, com índice de recidiva de $1,4 \%$. Arroyo et al ${ }^{13}$ realizaram o mesmo tipo de estudo, com 894 pacientes, também com resultados semelhantes, recidiva de $1,5 \%$.

Alguns autores referem que cerca de $1 \%$ dos submetidos à herniorrafia ambulatorial necessitarão hospitalização prolongada. ${ }^{14}$ Outros ampliam este número para aproximadamente $16 \% .{ }^{3}$ Dor na ferida operatória, náuseas e vômitos são as principais causas do prolongamento das internações desses pacientes. Num estudo em Hong $\mathrm{Kong}^{4}$, onde 271 herniorrafias foram realizadas ambulatorialmente, $98 \%$ dos pacientes receberam alta no dia da cirurgia. Apenas três pacientes permaneceram mais tempo internados por tontura, hiperglicemia e hemoptise. Em nosso estudo, nenhum dos pacientes submetidos ao procedimento ambulatorial necessitou de hospitalização maior que 24h. Apesar de preconizarem a realização da herniorrafia ambulatorial apenas em hérnias pequenas, em pacientes jovens e sem comorbidade, em nosso estudo, inclusive as hérnias primárias ínguino-escrotais grandes (Nyhus IIIB) foram operadas ambulatorialmente, sem desenvolvimento maior de complicações.

Em relação ao custo hospitalar, a herniorrafia ambulatorial representou, em nossa avaliação, uma economia média de $26,6 \%$ em relação a cada paciente internado. Deve ser também considerado que o sistema de alta precoce ou ambulatorial acarreta menor tempo de permanência hospitalar, maior rotatividade com maior número de ocupação e, conseqüente, redução da lista de espera para realização de herniorrafias. Anualmente são realizadas no CHSCPA cerca de 1400 herniorrafias. Se todas elas fossem realizadas pelo regime ambulatorial, ao final de um ano, a economia estimada para a instituição seria de $\mathrm{R} \$ 252.840,00$. 
Com efeito, nossos resultados reforçam a possibilidade e a alternativa da realização ambulatorial de procedimentos tradicionalmente eletivos, com necessidade de hospitalização e com conseqüentes listas de espera. Salientamos que as limitações de nosso estudo devem ser superadas com a expansão de nossa amostra o que também permitirá a verificação de outras variáveis.

Portanto, é evidente que, mesmo em um hospital escola, herniorrafias inguinais podem ser realizadas em nível ambulatorial sem que exista maior taxa de complicações que as previstas em qualquer hospital com internação convencional. Assim, o reparo de hérnia inguinal ambulatorial afirmase entre os cirurgiões como um tratamento seguro e de qualidade. Além disso, seu benefício financeiro é de tal relevância que contribui ainda mais para sua realização como rotina nas instituições de saúde. Ressaltamos, porém, que há necessidade de uma adaptação e organização em termos de políticas de saúde governamentais e institucionais para que a realização da herniorrafia inguinal ambulatorial seja integralmente instituída.

\begin{abstract}
Background: Ambulatory inguinal herniorraphy is performed worldwide. However, this procedure is not performed routinely in our reality. Our objetive is to analyze the benefits and complications of ambulatory inguinal herniorraphy in comparison to conventional intervention in our Service. Methods: A retrospective analysis was performed with 105 patients who uwderwent ambulatorial or conventional inguinal herniorraphy between February and October of 2002. Variables, including costs, were analyzed at the $15^{\text {th }}, 90^{\text {th }}$ and $180^{\text {th }}$ postoperative days. The Fischer's t test and chi-square test were used. Statistical significance was considered for a p value of p<0.05. Results: From 105 patients, seven were excluded. The total rate complication was $36.08 \%$. There was no significative statistical difference between groups at any time. The most common complication was local pain $(24.7 \%)$. The costs decreased $20 \%$ when surgery was performed in an ambulatory basis as well as the waiting time which was also reduced. Conclusions: The ambulatory herniorraphy is a safe procedure, decreases hospital stay, costs and it has a similar complication rate when compared to conventional herniorraphy.
\end{abstract}

Key words: Herniorraphy Local anesthesia; Inguinal hernia; Ambulatory surgical procedures; Complications.

\section{REFERÊNCIAS}

1. Kark AE, Kurzer MN, Belsham PA- Three thousand one hundred seventy-five primary inguinal hernia repairs: advantages of ambulatory open mesh repair using local anesthesia. J Am Coll Surg, 1998,186(4):447-456.

2. Miyazaki K, Nakamura F, Narita Y, et al. - Comparison of Bassini repair and mesh-plug repair for primary inguinal hernia: a retrospective study. Surg Today, 2001, 31(7):610-614.

3. Souza JAG, Silva A - Cirurgia Ambulatorial. Vol 1, Atheneu, Rio de Janeiro, 1999.

4. Lau H, Lee F, Poon J - Clinical factors influencing return to work after ambulatory inguinal herniorrhaphy in Hong Kong. Ambul Surg, 2001, 9(1):25-28.

5. Amid PK, Shulman AG, Lichtenstein IL - Local anesthesia for inguinal hernia repair step-by-step procedure. Ann Surg, 1994,220(6):735-737.

6. Pitrez FA - Pré e Pós- operatório em cirurgia geral e especializada. $2^{a}$ Edição. Porto Alegre. Artmed, 2003.

7. Zollinger RM - Hérnias. In Zollinger RM (ed) - Atlas de Cirurgia. $4^{\text {a }}$ ed. São Paulo. Artes Médicas, 1977, pp.348-359.

8. Diário Oficial da União, Portaria número 44/GM; 10 de janeiro de 2001.

9. Deysine M, Grimson RC, Soroff HS - Inguinal herniorrhaphy. Reduced morbidity by service standardization. Arch Surg; 126(5):628-630, 1991.
10. Doherty GM, Lowney JK, Mason JE, et al. - The Washington Manual of Surgery. $3^{\text {rd }}$ Edition. Philadelphia. Lippincott Williams \& Williams, 2002.

11. Boletim de Avaliação Interna Relativo à Análise e Produtividade do Bloco Cirúrgico do Hospital Santa Clara do Complexo Santa Casa/Ano 2002

12. Perez EM, Barriga R, Rodriguez MA, et al. - Ambulatory surgery for groin hernia: the Gilbert repair. Ambul Surg, 2000, 8(3):135138.

13. Arroyo A, Perez F, Ferrer R, et al. - Hernia surgery for the third millennium. Does classical herniorraphy still play a role? Ambul Surg, 2001, 9(2):73-75.

14. de Vooght A, Droissart R, Staudt JP, et al. - Open mesh plug hernioplasty in ambulatory surgery: a stud feasibility based on our experience in 413 procedures. Hernia, 2002, 6(3):108112.

Endereços para correspondência:

Daniel Nunes e Silva

Rua Guilherme Alves, 825/103

Bairro Jardim Botânico - Porto Alegre - RS

CEP 90680-001

Email: danielnunesesilva@terra.com.br 